\title{
Análise bibliométrica sobre o uso de aplicativos e tecnologias móveis no ensino dos processos de separação na Engenharia Química
}

Bibliometric analysis on use of apps and technologies in teaching separation processes at Chemical Engineering

Análisis bibliométrico sobre el uso de aplicaciones en tecnologías móviles en la enseñanza del procesos de separación en Ingeniería Química

Recebido: 06/08/2021 | Revisado: 15/08/2021 | Aceito: 19/08/2021 | Publicado: 22/08/2021

\author{
Paulo Victor da Silva Freitas \\ ORCID: https://orcid.org/0000-0002-3425-5744 \\ Universidade Federal do Triângulo Mineiro, Brasil \\ E-mail: paulo.freitas@uftm.edu.br \\ Kássia Graciele dos Santos \\ ORCID: https://orcid.org/0000-0001-7452-6900 \\ Universidade Federal do Triângulo Mineiro, Brasil \\ E-mail: kassia.santos@uftm.edu.br
}

\begin{abstract}
Resumo
O crescimento tecnológico desafia a sociedade na busca contínua por melhorias em seus processos, seja em atividades pessoais, econômicas ou industriais. É inegável o valor agregado que as tecnologias fornecem em nosso cotidiano. Contudo, uma análise de como obter o máximo proveito dessas ferramentas e suas possibilidades faz-se necessária. No âmbito do ensino, o uso de tecnologias digitais ainda se encontra em estágio embrionário, visto que muitas estruturas educacionais rejeitam o seu uso por não saberem como lidar com essas transformações para validar o aprendizado ou obter maior motivação e engajamento dos alunos. Dentro do contexto do ensino das Engenharias, principalmente em atividades de disciplinas que envolve projeto e cálculos, como as operações unitárias e processos de separação de misturas da Engenharia Química (assunto tão importante para o mercado de trabalho por compor os processos produtivos das indústrias químicas), é preciso reformular a estrutura teórica das universidades para melhor uso das ferramentas digitais, como ambientes de simulação, laboratórios virtuais e aplicativos móveis, em um ensino baseado em projetos, fomentando a proatividade e protagonismo dos alunos na busca pelo conhecimento, transformando assim o professor em um orientador, um guia durante esse processo.
\end{abstract}

Palavras-chave: Educação; Ensino; Operações unitárias; Simulação; Aplicativos móveis.

\begin{abstract}
The technological leverage challenges society to look for continuous improvement in their processes, whether in personal activities, economic or industrial. It is undeniable the aggregate value that technologies provide on a daily basis. In the teaching area, the use of digital technologies is still on an embryonic stage, since much of educational structures are rejecting its use because they do not know how to handle these transformations to validate the learning or to obtain students higher motivation and commitment. In the context of Engineering's learning, mainly in disciplines activities involving project and math calc, as unit operations and mixture separation processes of Chemical Engineering (subject with highly importance due to labor market and chemical industries' productive processes), it is necessary to reformulate the theoretical structure of Universities to do a better use of digital tools, as simulation environments, virtual labs and mobile applications, in a project-based learning scenario, fostering the students proactivity and protagonism in search for knowledge, to transform the teacher in an advisor, a guide during the processes.
\end{abstract}

Keywords: Education; Learning; Unit operation; Simulation; Mobile application.

\section{Resumen}

El crecimiento tecnológico desafía la sociedad en la búsqueda continua por mejoras en sus procesos, sea en las actividades personales, económicas o industriales. Es innegable el valor agregado que las tecnologías ofrecen en nuestro cotidiano. Todavía, un análisis de cómo obtener el máximo provecho de las herramientas y sus posibilidades se hace necesaria. En lo ámbito del enseñanza, el uso de tecnologías digitales se encontra en estágio embrionário, ya que muchas estructuras educacionales rechazan su uso por no saber cómo lidiar con esas transformaciones para validar el aprendizaje o obtenir maior motivación y compromiso de los alumnos. Dentro del contexto de la enseñanza de las Ingenierías, principalmente en actividades de las disciplinas que involucran proyectos y cálculos, como las operaciones unitarias y procesos de separación de mezclas en Ingeniería Química (tema en cuestión muy importante para el mercado de trabajo 
y para componer los procesos productivos de las industrias químicas), se necesita reformular las estructuras teóricas de las universidades para mejor uso de las herramientas digitales, cómo ambientes de simulación, laboratórios virtuales y aplicaciones móviles, en una enseñanza basada en proyectos, fomentando la proactividad y protagonismo de los alumnos en búsqueda del conocimiento, así transformando el profesor en un tutor, uno guía durante el proceso.

Palabras clave: Educación; Enseñanza; Operaciones unitarias; Simulación; Aplicaciones móviles.

\section{Introdução}

O crescente uso de dispositivos móveis muda toda a perspectiva de sociedade e, principalmente dentro de salas de aula, é um desafio para professores e toda a infraestrutura acadêmica vigente, pois faz-se necessário que o docente utilize metodologias avançadas de ensino, buscando o envolvimento do aluno com a tecnologia como um mecanismo educacional (Almeida e Santos, 2020; Almeida et al., 2020; Belarmino et al., 2020; Mangucci et al., 2020; Santos et al., 2021).

Porém, somente incluir as TICs no âmbito educacional não provê a inovação por si só, pois é necessário revisar a forma como a inclusão se dará, verificando-se as oportunidades que possam desafiar, tanto alunos quanto professores, utilizando seu potencial nos variados contextos de aprendizagem (Cruz et al., 2015).

A tecnologia deve ser um complemento à sala de aula, pensada principalmente - mas não somente - nas situações imprevisíveis, como por exemplo, a situação da pandemia do novo coronavírus em 2020, que fez com que um novo panorama fosse implantado às pressas pelos governos: A educação a distância no formato integral. Com isso, novas tecnologias e ferramentas tiveram que ser desenvolvidas e/ou melhoradas, para possibilitar a educação sem barreiras físicas. Porém, esse conceito de educação integral a distância ainda possui vários obstáculos no país, considerando que o Brasil possui grandes desigualdades sociais.

De acordo com os trabalhos de Christo et al. (2018) e Gomez et al. (2015), constata-se que um dos principais problemas enfrentados pelo ensino superior no Brasil é a alta taxa de evasão nas Universidades. Dentre os problemas que acarretam nas altas taxas de evasão em cursos relacionados à exatas, estão insatisfação com a estruturação curricular, baixo desempenho dos alunos bem como transferência de cursos/instituição (uma expectativa não alcançada pelo aluno ou que, pelas dificuldades enfrentadas, decide tentar outra carreira). Essas taxas altas de evasão em cursos de Engenharia giram em torno de $43 \%$.

Com sua pesquisa, Godoy (2017) verifica a necessidade de melhor adequação do currículo para receber a tecnologia de forma mais fácil e com todas as possibilidades que esta visa promover, como um coeficiente para se buscar uma menor evasão nos cursos de Engenharia (Figura 1). Para tanto, é preciso que o docente esteja sempre preparado e seja proativo na busca de recursos para prover soluções que sejam objetivas e que consigam ser motivadoras/inspiradoras para os alunos.

Neste contexto, o objetivo deste trabalho foi analisar o uso de tecnologias dentro do ambiente educacional, principalmente dentro das Engenharias e, especificamente, em uma área essencial para a indústria: as operações unitárias da Engenharia Química. Para tal, foi realizada uma revisão bibliométrica com base nas plataformas Scopus e Web of Science. Os dados foram mapeados e analisados empregando o software R Studio e a biblioteca Bibliometrix. Os resultados dos artigos de maior impacto foram então apresentados e discutidos. 
Figura 1: Melhorias necessárias no ensino de Engenharias através das TICS.

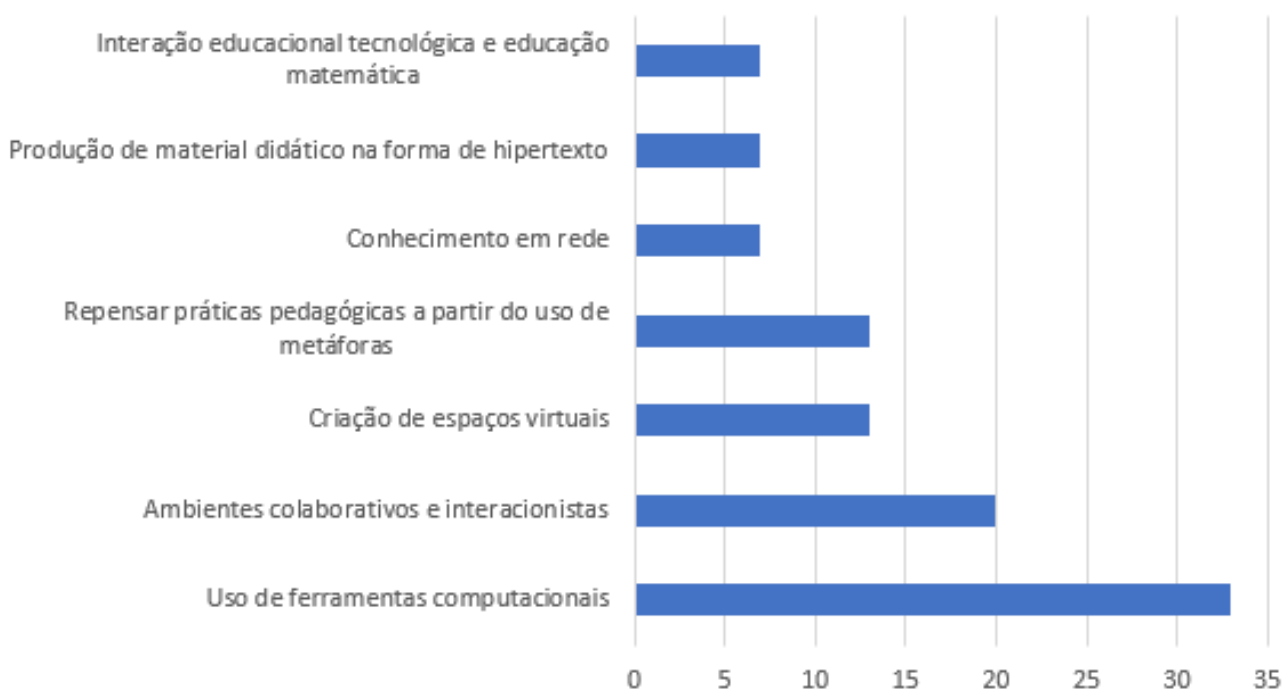

Fonte: Autores, adaptado de Godoy (2017).

\section{Metodologia}

De acordo com Köche (2009), a ciência é um processo de investigação em busca de uma relação existente entre os aspectos que envolvem os fatos, situações e acontecimentos, fenômenos ou coisas. Delimitar o problema a ser pesquisado, de acordo com o autor, é resultado de um trabalho mental, construção teórica que visa entender a malha de relações de interdependência que há entre os fatos. Portanto, o problema define os limites da dúvida, daquilo que deverá nortear a pesquisa e o trabalho da equipe.

Não obstante, a grande quantidade de publicações acadêmicas pode dificultar a análise bibliográfica e revisão de literatura por parte dos pesquisadores. Todavia, Aria e Curccurullo (2017) definem esse grande volume de publicações como crucial para utilização na base de conhecimento, sintetizando aquilo que já se conhece sobre a área de pesquisa e possibilitando oportunidades de inovação pelos pesquisadores.

Em seu trabalho, Aria e Curccurullo apontam também para a necessidade de se utilizar modelos bem estruturados para análise quantiativa de trabalhos científicos. Para isso, elencaram necessidades que podem ser respondidas através da análise bibliométrica, como a identificação da base de conhecimento do tópico a ser pesquisado e sua estrutura intelecutal, além de verificar os materiais científicos mais relevantes produzidos.

De modo a compreender o estado da arte do objeto de estudo proposto (uso de tecnologias educacionais nas engenharias no contexto de processos de separação), decidiu-se pelo uso da análise bibliométrica, por sua relevância no trato quantitativo e qualitativo dos dados elencados. Como apontado por Pereira et al. (2018), o enfoque matemático possibilita uma previsão de acontecimentos, e por isso um método quantitativo de análise auxilia no processo de dimensionamento do corpo da pesquisa.

Para esta análise bibliométrica, foram realizadas pesquisas sistematizadas nas plataformas Scopus e Web of Science, as quais são os bancos de dados de trabalhos científicos, artigos e periódicos com maior abrangência de áreas e que oferecem métricas que possibilitam uma análise por métricas. Para verificar a qualidade dos trabalhos elencados, uma análise qualitativa também foi realizada, com leitura dos artigos mais relevantes em cada etapa.

Todas as pesquisas foram realizadas buscando material entre as datas de 01/01/2015 até 21/09/2020 com o objetivo de analisar trabalhos mais atuais, com tecnologias mais avançadas, principalmente considerando o fato de que uma determinada ferramenta ou aplicação pode se tornar obsoleta em um curto período de tempo.

A Figura 2 apresenta as etapas da pesquisa, que foi feita inicialmente forma abrangente (Etapa 1), na qual foram 
investigados os temas Educação e Tecnologia. A Etapa 2 consistiu na busca dos temas Educação, Tecnologia e Engenharias, enquanto a Etapa 3 foi mais restritiva, ao buscar por Educação, Tecnologia, Engenharias e Processos de Separação. Porém, a fim de complementar o cenário brasileiro da Etapa 3, foi também realizada uma busca pelos termos em português, utilizando também a ferramenta de busca do Google Scholar.

Figura 2: Etapas de pesquisa.
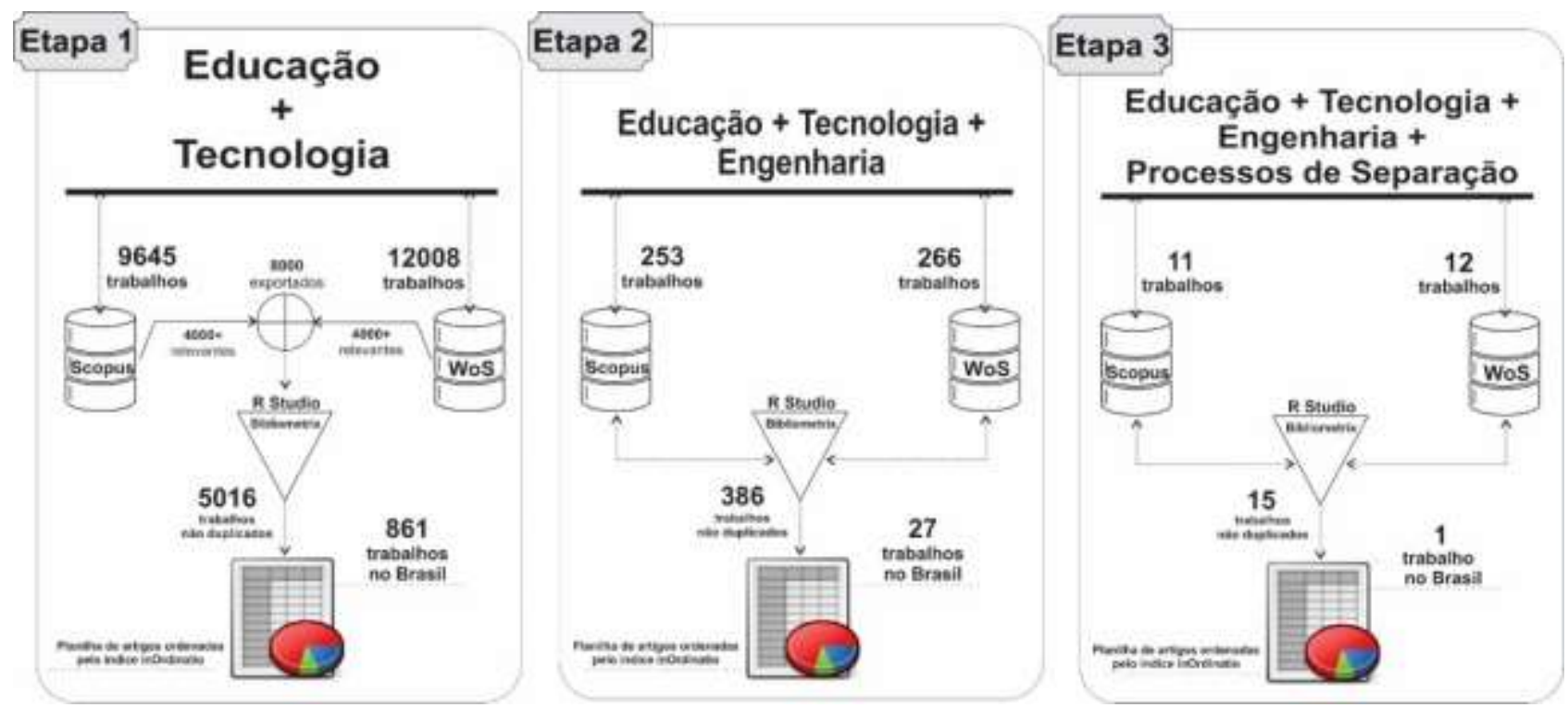

Fonte: Autores.

Utilizou-se do software R Studio juntamente com a biblioteca Bibliometrix (ARIA \& CUCCURULLO, 2017) para executar o mapeamento dos dados e análise através de índices disponibilizados pelos dois bancos de dados, facilitando a confecção de planilhas para seleção dos artigos a serem estudados. A pesquisa foi realizada em três etapas, cada uma com o seguinte fluxo:

a) Pesquisa por palavras-chave (keywords), sendo elas:

○ Keywords de Educação:

("teaching” OR “m-learning” OR “mobile learning" OR "distance learning" OR "classroom" AND "education")

- Keywords de Tecnologia:

("android" OR "ios" OR "hybrid" OR "software tool*" OR ("app" OR "apps" NOT "amyl*") OR "ict" OR "ubiqu*" OR "augmented" OR

"virtual" OR "simulat*")

○ Keywords de Engenharias:

(("aerospace" OR "computer" OR "industrial" OR "mechatronics" OR "civil" OR "electrical" OR "mechanical" OR "chemical") near/2 "engineer*")

- Keywords de Processos de separação:

("separation" OR "unit operation*")

b) Exportação de arquivos .csv das bases com índices para processamento

c) Geração de planilha de artigos agrupados, sem duplicidades, com dados das duas

bases de dados através do Bibliometrix. 
d) Busca por artigos e pesquisas realizadas dentro do tema no Brasil.

e) Separação dos artigos para leitura e análise (artigos internacionais e brasileiros) com base no fator inOrdinatio, disposto na Figura 3 (PAGANI et al., 2015; DE CARVALHO et al., 2020), que seleciona e ordena os trabalhos utilizando-se de um modelo multicritério (contagem de citações, ano de publicação e fator de impacto da revista).

Figura 3: Fórmula InOrdinatio aplicada sobre o fator de impacto dos artigos.

\begin{tabular}{|l|} 
Fórmula InOrdinatio $=\mathrm{FI}^{*} \mathrm{~A}+[10-(\text { AnoPesq }-\mathrm{AnoPub})]^{*} \mathrm{~B}+\left(\sum \mathrm{Ci}\right)^{*} \mathrm{C}$ \\
\hline $\mathrm{FI}=$ Fator de impacto \\
\hline AnoPesq = ano que a pesquisa está sendo realizada \\
\hline AnoPub = ano da publicação do paper \\
\hline $\mathrm{C} \mathrm{Ci}=$ número de citações \\
$\mathrm{A}=$ peso do critério $\mathrm{FI}$ (1 no InOrdinatio) \\
\hline $\mathrm{B}=$ nível de importância da contemporaneidade do tema, usualmente 1 a 10 \\
\hline $\mathrm{C}=$ Peso do Critério Citações (1 no inOrdinatio) \\
\hline
\end{tabular}

Fonte: Autores.

\section{Resultados e Discussão}

\subsection{Etapa 1: Busca pelos temas "educação" e "tecnologia"}

Para a Etapa 1, a busca na base da Scopus e Web of Science retornaram, respectivamente, 9.645 e 12.008 trabalhos. Considerando os dados brutos levantados, verificou-se que os países líderes em pesquisas na área de tecnologia e educação são Estados Unidos e Espanha, como mostra a Figura 4.

Figura 4: Países que mais publicaram no tema Educação e Tecnologia, de acordo com as plataformas:

(a) Scopus; (b) Web of Science.

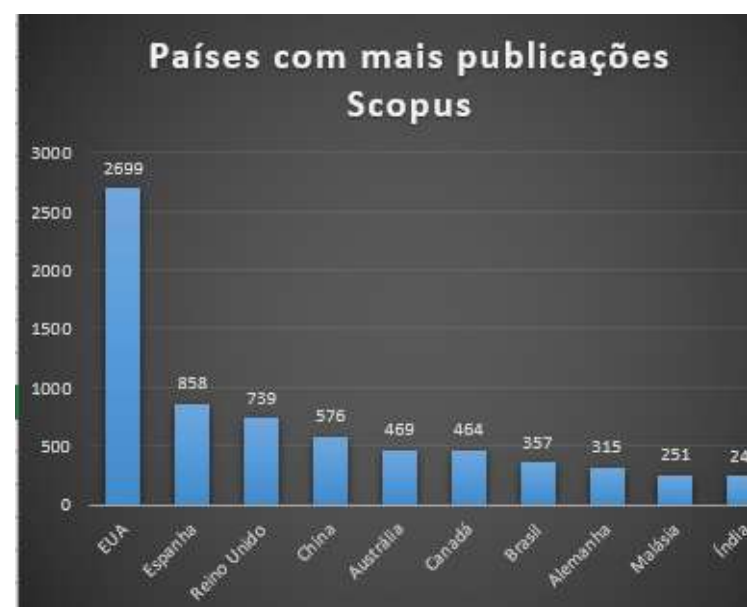

(a)

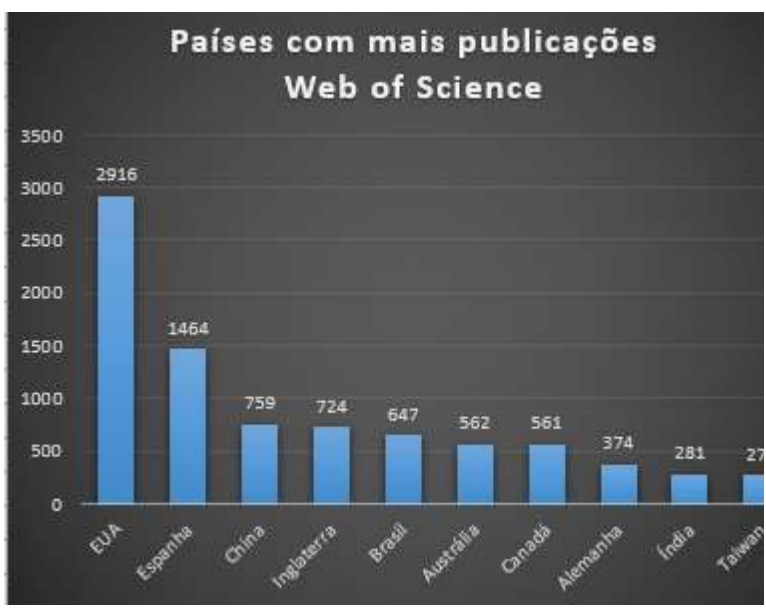

(b)

Fonte: Autores.

Observa-se na Figura 4 que o Brasil está entre a $5^{\text {a }}$ posição (pela plataforma Web of Science) e a $6^{a}$ posição (plataforma Scopus). Válido ressaltar que as pesquisas podem possuir múltiplas colaborações, considerando-se que um pesquisador desenvolve e auxilia a pesquisa de outros pesquisadores fora de seu país, fortalecendo assim a corrente científica. A Figura 5 apresenta as maiores redes de contribuição visualizadas nos artigos pesquisados, que englobam os pesquisadores de países como Estados Unidos, Espanha, China e Reino Unido. Enquanto os EUA, líder nas pesquisas, apresenta uma interação diversificada, trabalhando em cooperação com vários países, o Brasil tem apresentado uma pequena cooperação internacional nesta área de pesquisa. 
Para realizar o estudo bibliométrico pelo Bibliometrix ${ }_{2}$ decidiu-se pela exportação de um total de 8.000 artigos (4.000 artigos em cada, considerando a ordenação padrão das bases por RELEVÂNCIA). Ao agrupar os dados exportados utilizando o software R Studio, gerou-se uma planilha com 5.016 artigos não duplicados.

Figura 5: Mapa da colaboração em pesquisa entre países, na área de Educação e Tecnologias.

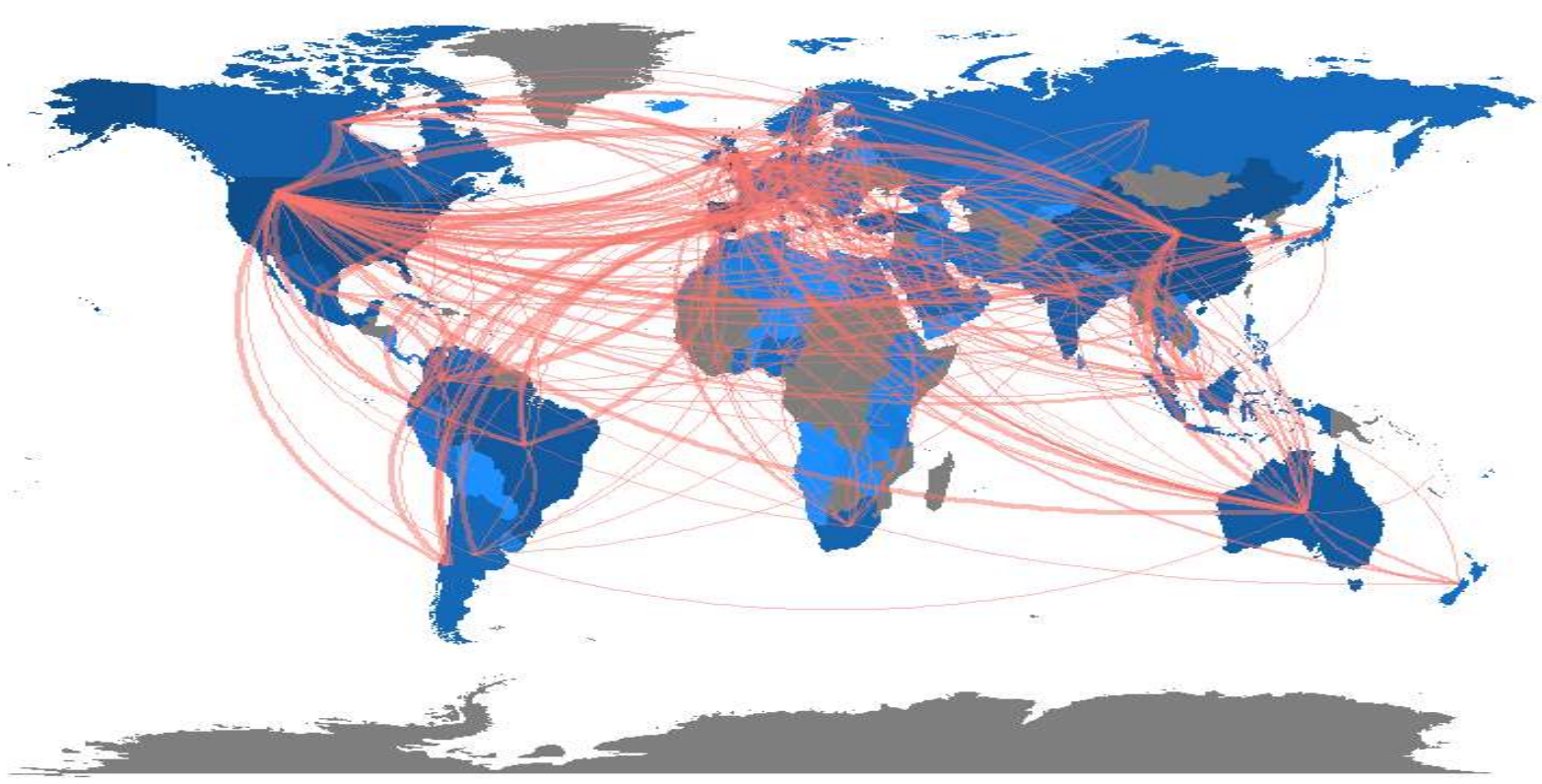

Fonte: Autores, utilizando o Bibliometrix.

Ao verificar o resultado gerado na planilha, observa-se que o número de pesquisas entre 2015 e 2020 sobre o assunto, é crescente (Figura 6), mostrando a importância da utilização de tecnologias, aplicativos e simulações dentro do ambiente educacional, o que corrobora com a importância abordada na seção de introdução, principalmente pela atual facilidade de acesso à equipamentos e conectividade.

Importante também ressaltar a situação de pesquisa nacional sobre o tema. O Brasil possui 861 trabalhos no tema de tecnologias e aplicativos voltados para a educação, o que demonstra que o cenário nacional é relativamente impactado e interessado em pesquisar e desenvolver novos produtos nesse quesito.

Apesar da Figura 6 mostrar um decréscimo de pesquisas realizadas em 2020, vale ressaltar que o levantamento considerou até o mês de Setembro e não a totalidade do ano. Também cabe avaliação do impacto da pandemia do novo coronavírus nas pesquisas referentes à educação, pois, além um enorme período de quarentena mundial que afetou a produção científica, grande parte das publicações até o momento estão voltadas para soluções de combate ao vírus. Contudo, a quantidade de pesquisas na área de tecnologia e educação continua relevante. 
Figura 6: Número de publicações sobre o tema através dos anos.

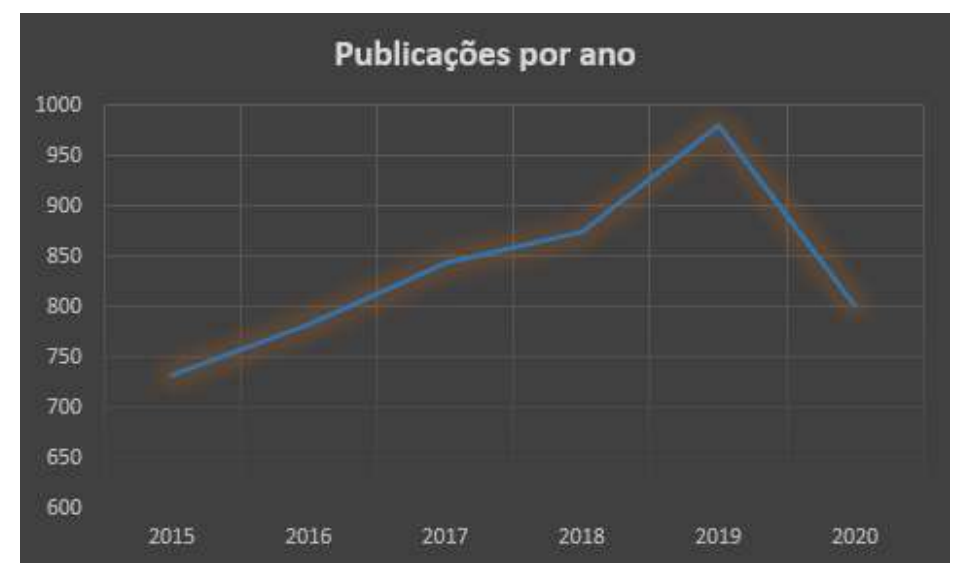

Fonte: Autores.

Analisando os principais termos encontrados nos artigos, temos o seguinte mapa (Figura 7) indicando uma clara atenção dada aos pesquisadores na educação e no modo como essas novas tecnologias afetam os estudantes e as formas de se ensinar.

Além disso, verifica-se também a menção a tecnologias que são mais utilizadas no contexto, como simulação, realidade virtual e aumentada, além de abordagens com dispositivos móveis (em menor escala).

Figura 7: Termos mais comuns nos artigos.

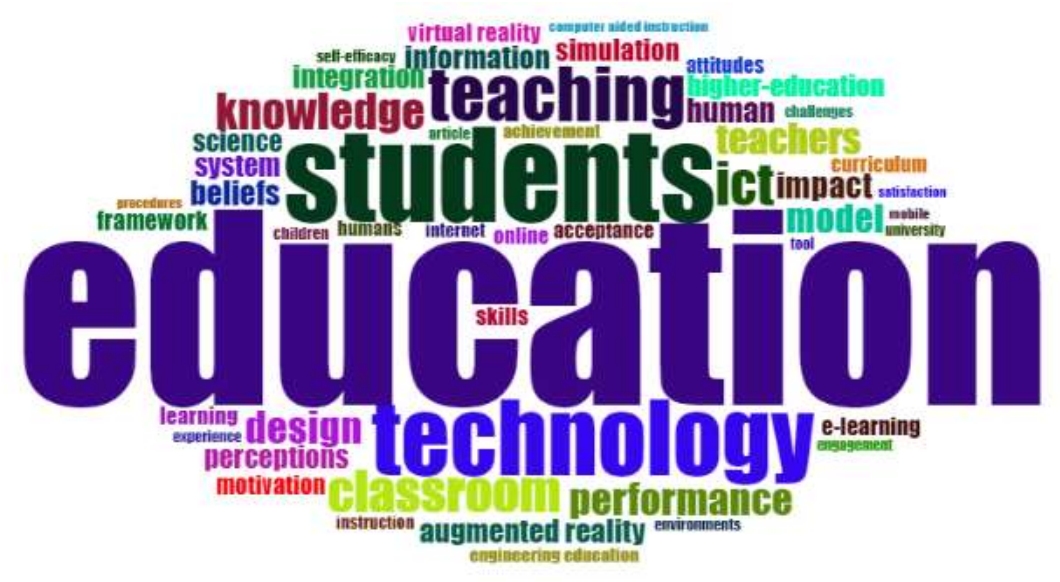

Fonte: Autores, utilizando o Bibliometrix.

Após essa primeira revisão métrica, os artigos foram analisados pelo método inOrdinatio (Figura 3) e consequentemente, os de maior relevância foram selecionados através de análise textual para verificar a coerência do trabalho com o tema da revisão proposta.

A Tabela 1 mostra os cinco artigos internacionais e nacionais mais citados na busca realizada na Etapa 1, que mostra de forma geral o emprego de novas tecnologias na área da educação. Os artigos relacionados demonstram que o impacto do uso de dispositivos tecnológicos dentro de ambientes educacionais ocorre principalmente em duas vertentes: dispositivos móveis e ambientes virtuais de aprendizagem, como laboratórios virtuais e simulações. 
Tabela 1: Artigos Internacionais e Nacionais mais relevantes segundo método inOrdinatio, para a Etapa 1.

\begin{tabular}{|c|c|c|c|}
\hline Ordem & Autor & Título & \\
\hline $1^{\mathrm{o}}$ & SUNG et al. & $\begin{array}{l}\text { The effects of integrating mobile devices with teaching and learning on } \\
\text { students learning performance: a meta-analysis and research synthesis }\end{array}$ & \multirow{5}{*}{ Internacionais } \\
\hline $2^{\circ}$ & MARTÍN-GUTIÉRREZ et al. & Virtual technologies trends in education & \\
\hline $3^{\circ}$ & POTKONJAK et al. & $\begin{array}{l}\text { Virtual laboratories for education in science technology and } \\
\text { engineering: a review }\end{array}$ & \\
\hline $4^{\circ}$ & HERADIO et al. & Virtual and remote labs in education: a bibliometric analysis & \\
\hline $5^{\circ}$ & ZYDNEY et al. & Mobile apps for science learning: review of research & \\
\hline $1^{\circ}$ & DE FARIA et al. & $\begin{array}{l}\text { Virtual and stereoscopic anatomy when virtual reality meets medical } \\
\text { education }\end{array}$ & \multirow{5}{*}{ Nacionais } \\
\hline $2^{\circ}$ & BARATA et al. & $\begin{array}{l}\text { Consolidating learning in power systems virtual reality applied to the } \\
\text { study of the operation of electric power transformers }\end{array}$ & \\
\hline $3^{\circ}$ & DE SOUSA MONTEIRO et al. & Youubi: open software for ubiquitous learning & \\
\hline $4^{\circ}$ & VIEGAS et al. & Impact of a remote lab on teaching practices and student learning & \\
\hline $5^{\circ}$ & MORAES et al. & $\begin{array}{l}\text { Integrating mobile phones into science teaching to help students } \\
\text { develop a procedure to evaluate the corrosion rate of iron in simulated } \\
\text { seawater }\end{array}$ & \\
\hline
\end{tabular}

Fonte: Autores.

Sung et al. (2016) e Zydney et al. (2016) realizaram revisões bibliográficas buscando um delineamento sobre a forma como as tecnologias móveis devem ser utilizadas, destacando a possibilidade multidisciplinar de sua adoção, além de definirem a importância de novos estudos quantitativos sobre o tema e a necessidade do envolvimento da infraestrutura acadêmica e do professor na forma de lidar com a tecnologia em sala de aula.

Os trabalhos de Moraes et al. (2015) e De Sousa Monteiro et al. (2016) confirmaram as ideias e necessidade de novos estudos abordados por Sung e Zydney, ao explorarem na prática aplicações móveis no contexto de ciências, em que o uso do celular supre a demanda por equipamentos caros e específicos no estudo do processo de avaliação de corrosão do ferro em água do mar e de aprendizado ubíquo (aplicação Youubi), respectivamente.

Heradio et al. (2016) executaram uma revisão bibliométrica utilizando as bases Scopus, Web of Science e GRC 2014, utilizando abordagem de mapping science e análise de performance, de modo a verificarem a maturidade do uso de laboratórios virtuais no cenário educacional.

Potkonjak et al. (2016) apresentaram uma revisão crítica sobre conceitos e tecnologias empregadas no campo de laboratórios baseados em software para verificação das demandas da área e provimento de campo para futuros estudos.

Martín-Gutiérrez et al. (2017) realizaram um estudo do histórico da tecnologia baseada em realidade virtual, indicando seus modos de utilização, bem como analisando fatores de custo/benefício de sua adoção na vertente educacional.

Barata et al. (2015) utilizaram um modelo computacional para estudos da operação de transformadores de energia elétrica na Universidade do Pará, enquanto Viegas et al. (2016) utilizaram o VISIR (laboratório virtual) na PUC-Rio. Tais estudos analisaram os índices de aceitação dos estudantes, facilidade na retenção dos conhecimentos, bem como a possibilidade de cooperação entre os alunos. 
De Faria et al. (2016) demonstraram como a utilização de imagens geradas por software podem auxiliar no ensino de neuroanatomia, através de uma pesquisa de satisfação após utilizar grupos distintos de alunos, nos quais o primeiro grupo continuava com a prática tradicional de ensino, e os grupos 2 e 3 possuíam abordagem de fotos com e sem estereoscopia, respectivamente.

\subsection{Etapa 2: Busca pelos temas "educação", "tecnologia e "engenharias"}

Para a $2^{\mathrm{a}}$ etapa de pesquisas, toda a etapa 1 foi replicada, porém utilizou-se também palavras-chave que remetem às áreas de engenharia, como uma forma de se verificar o estado da arte das pesquisas envolvendo tecnologia e educação nesses campos.

Como resultado da pesquisa, as plataformas Scopus e Web of Science obteve-se, respectivamente, 253 e 266 trabalhos. Analisando novamente os dados brutos (sem exclusão de duplicações), verificamos que os países que mais contribuíram para pesquisas são Estados Unidos e Espanha.

O Brasil aparece com destaque na terceira posição nas duas bases, mostrando que as engenharias são pólo científico importante para o país, de acordo com a Figura 8.

Ressalta-se que, embora a busca sistematizada da Etapa 2 não tenha considerado os artigos já analisados de MartínGutiérrez et al. (2017), Potkonjak et al. (2016), Heradio et al. (2016), Barata et al. (2015) e Viegas et al. (2018), estes deveriam aparecer na etapa 2 por terem como objetos principais de estudo os efeitos de laboratórios virtuais na educação (voltados para engenharias), porém esses não foram considerados e nem contabilizados para efeito de análise.

Figura 8: Países que mais publicaram no tema Educação, Tecnologia e Engenharias (Etapa 2), nas plataformas (a) Scopus; (b) Web of Science.

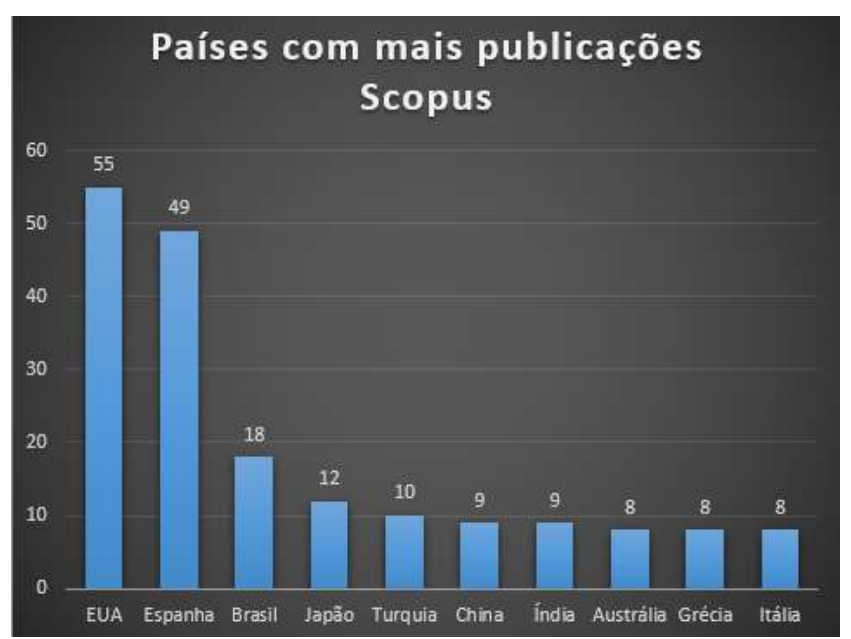

(a)

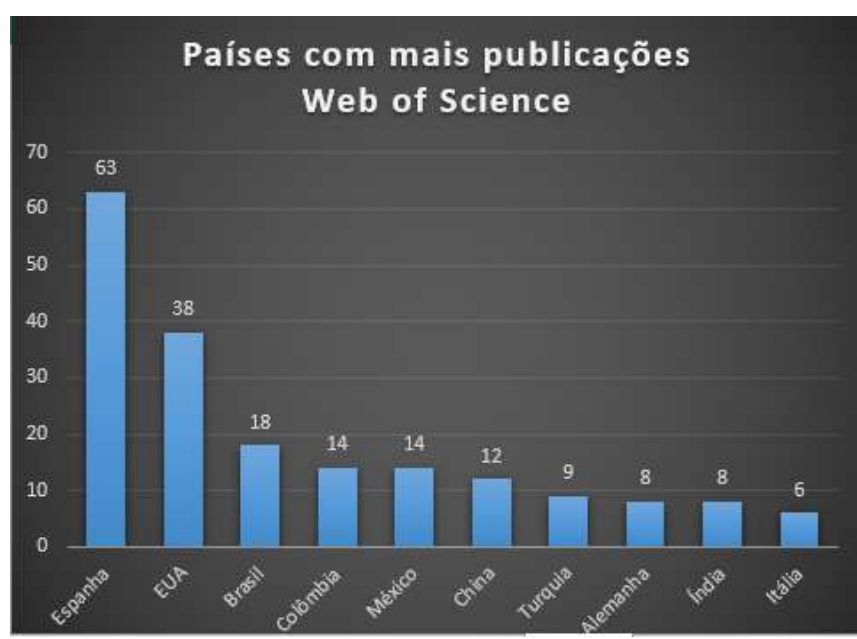

(b)

Fonte: Autores.

Apesar do Brasil se destacar entre os países que mais pesquisam sobre o tema, uma análise do mapa de colaboração internacional mostra que o país possui uma única linha de colaboração (França), algo que não acontece com Estados Unidos e Espanha, que possuem colaboração global em pesquisas sobre esse assunto, como demonstra a Figura 9. Esse dado brasileiro pode ser negativo quando analisado o aspecto de troca de experiências e transferência tecnológica global. 
Figura 9: Mapas de colaboração em pesquisa, no tema Educação, Tecnologia e Engenharias (Etapa 2):

(a)todos os países; (b)interação do Brasil com outros países.

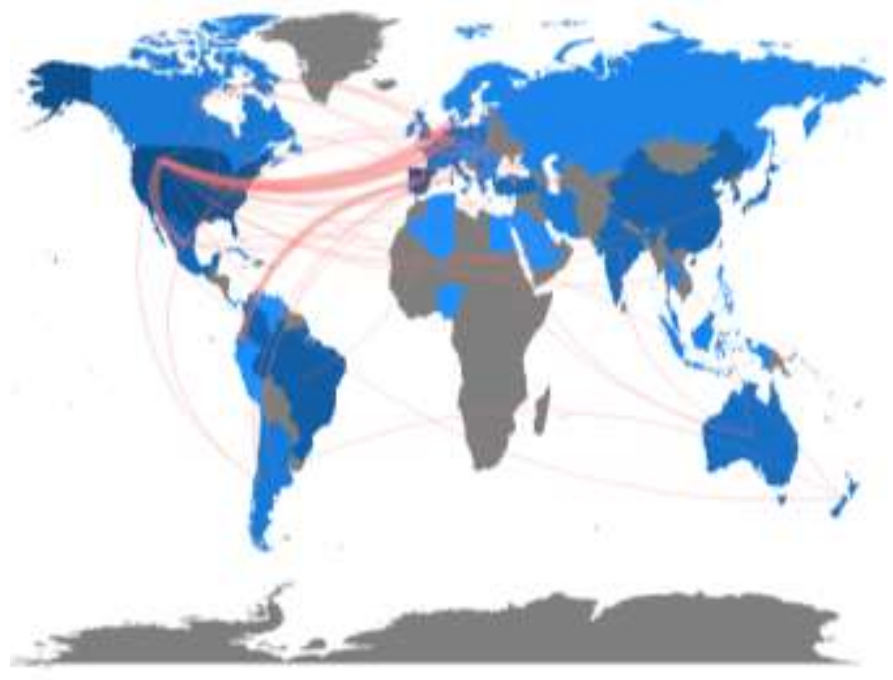

(a)

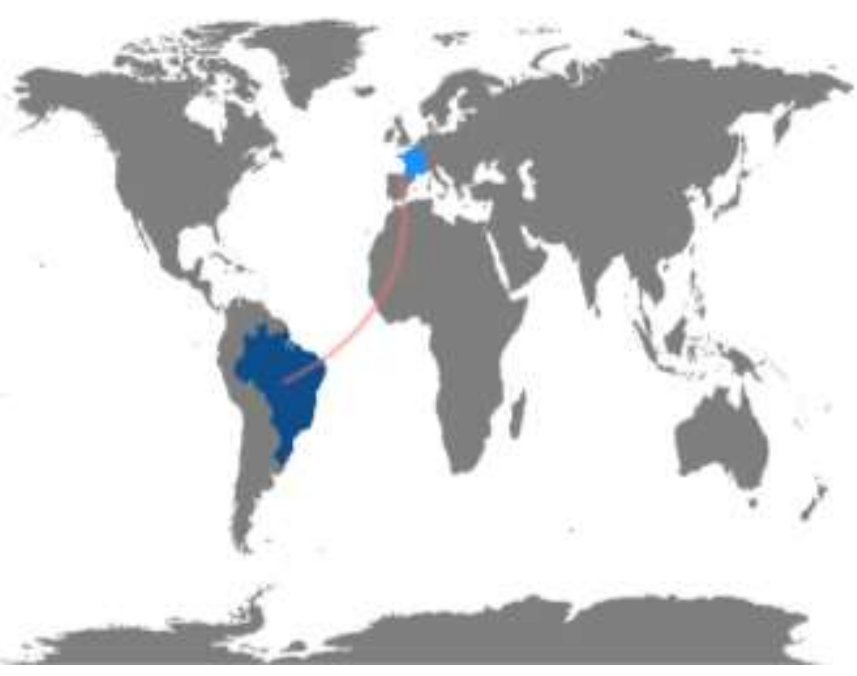

(b)

Fonte: Autores, utilizando o Bibliometrix.

Após o processamento dos dados utilizando o Bibliometrix, obteve-se 386 trabalhos dentro da área, sendo 27 trabalhos nacionais. Verificou-se, através dos dados da Figura 10, que o número de publicações por ano se manteve crescente, embora em 2017 tenha havido uma queda na quantidade de pesquisas submetidas. Mesmo com a situação da pandemia de 2020, o número de publicações mostra um aumento, indicando que o número de pesquisas no tema (tecnologias na educação no campo das engenharias) pode atingir o pico, ultrapassando o quantitativo verificado em 2019.

Figura 10: Publicações sobre o tema através dos anos, para a Etapa 2.

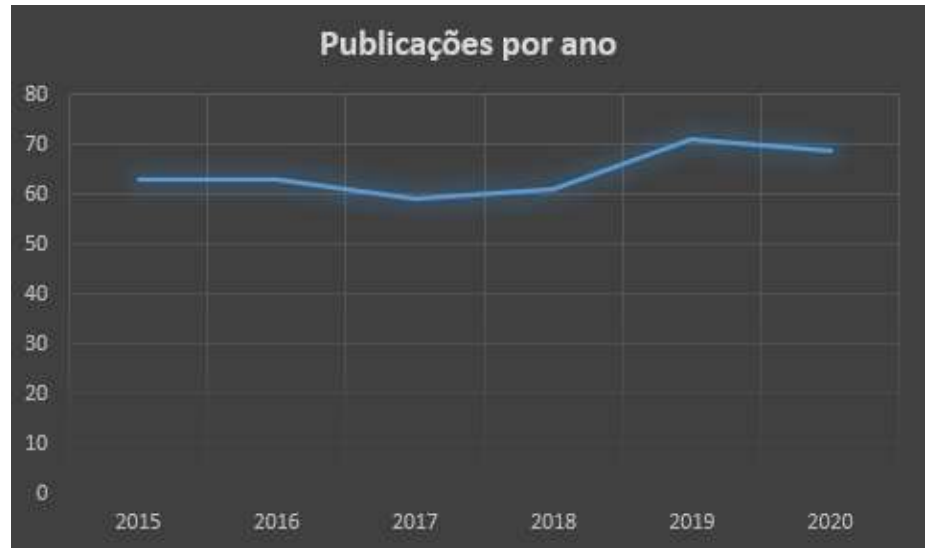

Fonte: Autores.

No campo semântico, observa-se uma similaridade de termos com os da Etapa 1. No entanto, tem-se a presença de termos que indica uma necessidade de reestruturação ou revisão curricular devido ao uso de tecnologias e aplicativos nas engenharias (curricula), além de termos que sinalizam para outras necessidades vinculadas com a área, como performance, laboratórios (virtuais) e modelos computacionais (Figura 11). 
Figura 11: Termos mais comuns nos artigos abordados na Etapa 2.

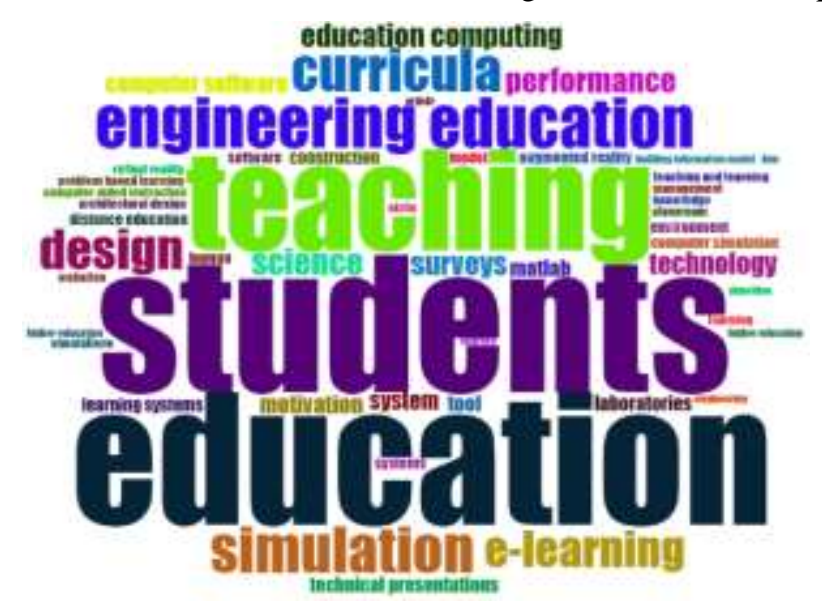

Fonte: Autores, utilizando o Bibliometrix.

Após essa análise, os artigos processados (sem duplicação) foram ordenados novamente pelo fator inOrdinatio (Figura 7) e selecionados de acordo com a meta da análise bibliométrica:

Tabela 2: Artigos Internacionais e Nacionais mais relevantes segundo método inOrdinatio (Etapa 2).

\begin{tabular}{|c|c|c|c|}
\hline Ordem & Autor & Título & \\
\hline $1^{\circ}$ & MARTÍN-GUTIÉRREZ et al. & $\begin{array}{l}\text { Augmented reality to promote collaborative and autonomous learning } \\
\text { in higher education }\end{array}$ & \multirow{5}{*}{ Internacionais } \\
\hline $2^{\circ}$ & QU, X et al. & $\begin{array}{l}\text { An improved TLBO based memetic algorithm for aerodynamic shape } \\
\text { optimization }\end{array}$ & \\
\hline $3^{\circ}$ & JUSKEVICIENE et al. & $\begin{array}{l}\text { Application of 1:1 mobile learning scenarios in computer engineering } \\
\text { education }\end{array}$ & \\
\hline $4^{\circ}$ & GARCIA-ZUBIA et al. & $\begin{array}{l}\text { Empirical analysis of the use of the VISIR remote lab in teaching } \\
\text { analog electronics }\end{array}$ & \\
\hline $5^{\circ}$ & LI et al. & $\begin{array}{l}\text { An inverted classroom approach to educate MATLAB in chemical } \\
\text { process control }\end{array}$ & \\
\hline $1^{\circ}$ & BOTELHO et al. & $\begin{array}{l}\text { Kolb's experiential learning theory and Belhot's learning cycle guiding } \\
\text { the use of computer simulation in engineering education a pedagogical } \\
\text { proposal to shift toward an experiential pedagogy }\end{array}$ & \multirow{5}{*}{ Nacionais } \\
\hline $2^{\circ}$ & HENRIQUE et al. & Optimization of chemical engineering problems with EMSO software & \\
\hline $3^{\circ}$ & BOAROLI et al. & $\begin{array}{l}\text { Data monitoring and hardware control for app android by Bluetooth } \\
\text { communication for laboratory teaching in electrical engineering } \\
\text { courses }\end{array}$ & \\
\hline $4^{\circ}$ & MESQUITA et al. & The skateboard factory: a teaching case on discrete-event simulation & \\
\hline $5^{\circ}$ & SOTELINO et al. & Teaching BIM and its impact on young professionals & \\
\hline
\end{tabular}

Fonte: Autores.

Martín-Gutiérrez et al. (2015) descreveram sobre o desenvolvimento de ferramentas de realidade aumentada em disciplinas da Engenharia Elétrica com foco em facilitar o compartilhamento de experiências e fomentando uma forma de ensino 
mais autônomo.

Qu et al. (2017) demonstraram a criação de um algoritmo para otimizar formas aerodinâmicas dentro da Engenharia Aeroespacial. O algoritmo criado foi validado e testado em softwares de simulação.

Juskeviciene et al. (2016) realizaram um questionário online com professores da Lituânia para verificar o cenário do aprendizado móvel personalizado em Engenharia da Computação e outras disciplinas de ciências, matemáticas e engenharias, como forma de aprimorar a abordagem tecnológica nas universidades.

Garcia-Zubia et al. (2016) aplicaram testes em diversas turmas, de disciplinas e países diferentes como forma de avaliar o impacto do uso do VISIR (laboratório remoto) no ensino básico de eletrônica.

Li et al. (2017) propuseram uma abordagem acadêmica para salas de aula invertida (flipped classroom) em Engenharia Química no ensino de habilidades em programação MatLab para simular modelos de equação diferencial ordinária, executar a Transformada de Laplace e o design de controladores PID para processos químicos.

Botelho et al. (2016) projetaram um ambiente e bases pedagógicas baseadas na experimentação de Kolb e o ciclo de Beholt para utilização otimizada da simulação nos cursos de Engenharia utilizando como modelo o software RoboMind (robô virtual).

Henrique et al. (2018) demonstraram a versatilidade do software EMSO, comumente utilizado como um simulador de processo orientado a equações, mas que no estudo dos autores transformou-se em uma ferramenta para a otimização e solução de problemas complexos de Engenharia Química.

Boaroli et al. (2017) desenvolveram um componente com fins didáticos em arduino para gerenciamento de sistemas eletrônicos através de bluetooth. Esse dispositivo tornou possível medir variáveis e controlar o sistema desenvolvido em laboratório, sem a necessidade de um ambiente específico e possibilitando o uso durante a aula teórica.

Mesquita et al. (2017) avaliaram em seu trabalho o desenvolvimento de um objeto em simulação computacional, no qual os alunos de Engenharia de Produção passaram por todas as etapas de manufatura e concepção do produto virtual.

Sotelino et al. (2020) estudaram o impacto do ensino de Modelagem da Informação em Construções em profissionais jovens, utilizando de softwares para fortalecer os conceitos e facilitar a comunicação entre equipes.

\subsection{Etapa 3: Busca pelos temas "educação", "tecnologia", "engenharias" e "processos de separação"}

Para finalizar a análise bibliométrica proposta, a terceira etapa contou com a adição de termos voltados para o ensino de operações unitárias (área da Engenharia Química) às palavras-chave anteriormente utilizadas, com a tentativa de verificar a situação das pesquisas e desenvolvimento de produtos nesse campo específico.

A Etapa 3 retornou uma quantidade menor de trabalhos (15 trabalhos não duplicados), mostrando que há espaço para desenvolver pesquisas nesse tema, em simulação, laboratórios virtuais, aplicações para dispositivos móveis e ambientes educacionais aplicados às operações unitárias da indústria.

Entre os países que publicaram artigos sobre o tema de 2015 até os dias atuais, estão Espanha (6), Hungria (2), China, Dinamarca, Brasil, Portugal, Reino Unido, Irã e Emirados Árabes (1 artigo cada).

A Tabela 3 mostra a lista de artigos encontrados nas bases pesquisadas, ordenadas pelo fator inOrdinatio. 
Tabela 3: Artigos Internacionais e Nacionais mais relevantes segundo método inOrdinatio (Etapa 3, que contabiliza os temas Educação, Tecnologias, Engenharias e Processos de Separação).

\begin{tabular}{|c|c|c|c|}
\hline Ordem & Autor & Título & \\
\hline $1^{\mathrm{o}}$ & CALVO et al. & $\begin{array}{l}\text { The teaching of enhanced distillation processes using a commercial } \\
\text { simulator and a project-based learning approach }\end{array}$ & \multirow{5}{*}{ Internacionais } \\
\hline $2^{\circ}$ & RODRIGUEZ et al. & $\begin{array}{l}\text { Motivational active learning: an integrated approach to teaching and } \\
\text { learning process control }\end{array}$ & \\
\hline $3^{\circ}$ & ROMAN et al. & $\begin{array}{l}\text { Achieving a better understanding of binary azeotropic mixtures } \\
\text { distillation through aspen plus process simulations }\end{array}$ & \\
\hline $4^{\circ}$ & UDUGAMA et al. & $\begin{array}{l}\text { A novel use for an old problem the Tennessee Eastman challenge process } \\
\text { as an activating teaching tool }\end{array}$ & \\
\hline $5^{\circ}$ & BORREGUERO et al. & Simulator-based learning in the teaching of chemical engineering & \\
\hline $1^{\mathrm{o}}$ & FREGOLENTE et al. & $\begin{array}{l}\text { Project based learning applied to distillation and absorption education } \\
\text { integration between industry and a chemical engineering undergraduate } \\
\text { course }\end{array}$ & Nacional \\
\hline
\end{tabular}

Fonte: Autores.

Como objetivo de seu trabalho, Calvo et al. (2016) analisaram a influência do uso do simulador Aspen Plus em um curso voltado para os processos de separação aprimorados, necessários para separar substâncias azeotrópicas. O curso ministrado na Universidad Complutense de Madrid era composto de aulas sobre o tema, seminários e aulas de laboratório, nos quais os alunos simularam exemplos das obras e também casos reais da indústria.

Após estas simulações, os alunos apresentavam um relatório crítico de suas análises, como uma forma de melhorar a retenção do que foi estudado e simulado, evitando que o aluno simplesmente utilizasse dados aleatórios para resolução.

Assim como Calvo et al. (2016), Rodriguez et al. (2018) também descreveram o impacto educacional de uma abordagem diferenciada no ensino, com a adoção da tecnologia. No caso desse segundo trabalho, utilizou-se um modelo de sala de aula invertida no ensino de Processos de Controle, no qual os alunos são responsáveis pelo controle estratégico de diferentes equipamentos encontrados na indústria (reatores, compressores, colunas de destilação, entre outras operações unitárias). Inicialmente, os professores disponibilizaram vídeos e materiais complementares. Após estudar esse material, os alunos encontravam-se com os docentes e respondiam questionários online sobre o conteúdo, além de passarem por atividades baseadas em gamificação. Depois, efetuavam a simulação dos conceitos no software de simulação (Aspen Dynamics com dados via Excel + Visual Basic).

Roman et al. (2019) exploraram também o uso de simulador (Aspen Plus) na retenção de conhecimento pelos alunos. No trabalho, os autores criticam as abordagens de outros estudos, que indicam a satisfação do aluno somente por meio de questionários, detalhando pouco o uso do simulador ou falta da descrição dos problemas analisados. Com isso, elaboraram casos de uso que foram exemplificados no artigo, como uma forma de visualizar melhor o uso e benefícios da abordagem por simulação (três casos, sendo eles: Destilação binária simples (metanol + água), por oscilação de pressão (etanol + benzeno) e heterogên ea (água + 1-butanol). Os autores indicaram principalmente a possibilidade e facilidade de se manusear variáveis como o principal ponto na adoção de simuladores dentro da sala de aula, visando preparar o aluno para as mais diversas situações da indústria.

Udugama et al. (2020) utilizaram em seu trabalho o Tennessee Eastman (software de benchmark para controle de processo) como uma ferramenta para criação de plantas de processo de controle que, além de simular, oferecem ao discente um 
ambiente onde as implicações matemáticas não ficam ocultas. A partir da parte gráfica do TEP, os alunos podem entender melhor como funcionam operações complexas e tarefas de controle dentro de um ambiente real, além dos controles hierárquicos e interdependência entre os fluxos e equipamentos do processo. Os autores verificaram que o TEP oferece uma excelente plataforma de ensino com múltiplas oportunidades, como: apresentar aos alunos a dinâmica de um processo realista, proporcionar a alteração em tempo real de fatores, fornecer a possibilidade de se trabalhar em um único problema durante um semestre com continuidade, aumento progressivo de dificuldade do problema de controle enfrentado.

Borreguero et al. (2019) efetuaram comparações de resultados entre o software Aspen Plus e Aspen HYSYS em um projeto de produção de ácido sulfúrico junto aos alunos de último ano de Engenharia Química da Universidade de Castilla. Os resultados do trabalho mostraram um bom engajamento e trabalho cooperativo dos alunos, concluindo assim que o uso de programas de simulação explorando casos reais da indústria é um modo atrativo de introduzir os processos industriais e simuladores comerciais para os discentes. Ressalta-se que os resultados dos processamentos dos softwares foram similares.

Fregolente et al. (2018) utilizaram o Aspen Plus na disciplina de Operações Unitárias de Transferência Difusional, dentro de uma perspectiva de ensino baseado em projetos, os alunos são motivados a resolverem problemas reais de indústrias de polpa através da construção de plantas virtuais e apresentação de trabalhos em forma de palestras. A abordagem proposta pelos autores mistura conceitos teóricos e práticos que aproximam o discente do objeto do conhecimento.

Com o intuito de aprofundar a situação do ensino de operações unitárias e processos de separação no Brasil por meio de tecnologias, uma pesquisa complementar foi realizada utilizando o Google Scholar como ferramenta de buscas. Importante salientar que a busca teve como parâmetros as datas de 01/01/2015 até 24/09/2020, com os termos em português que remetem a educação, tecnologia e processos de separação (ensino or educação or "engenharia química" or "separação" or "operaçães unitárias" or aplicativo por "app" or "software" or "android" or "iOS" or "hibrido").

A ferramenta do Google busca por trabalhos em bases externas, porém seu mecanismo de pesquisa é menos completo do que as ferramentas de busca da Scopus e Web of Science. Além de não retornar os dados com métricas suficientes para análises bibliométricas, o buscador não possui uma ferramenta de seleção de tipos de documentos, retornando capítulos e indicações de livros sobre o tema (o que não será considerado no estudo).

Como resultado da busca pelos termos citados, obteve-se 82 resultados, que foram exportados para uma planilha. Após isso, selecionou-se os tipos de documentos, sendo que somente artigos e teses foram analisados. Em seguida, verificou-se a relação entre a pesquisa e o tema do trabalho. Ao final da seleção, obteve-se uma lista de 5 trabalhos, dispostos na Tabela 4, de acordo com a quantidade de citações (inOrdinatio não foi utilizado pela falta de métricas decorrentes da exportação de bases de dados da Google Scholar).

Lima (2019) desenvolveu o aplicativo híbrido PEIQ (Projetando Equipamentos da Indústria Química) durante o Programa de Mestrado em Inovação Tecnológica da Universidade Federal do Triângulo Mineiro - Campus Uberaba. Esse aplicativo auxilia no processo de dimensionamento de equipamentos de separação sólido-fluido nos campos gravitacionais e centrífugo. O PEIQ é um sistema que possui módulos para os seguintes equipamentos da indústria química: Câmara de poeira, Centrífuga, Ciclone, Elutriador, Hidrociclone e Sedimentador. Os módulos baseiam-se em contribuições de diversos autores sobre o tema, entre eles Cremasco, Massarani, Peçanha, Svarovsky, Geankoplis e Castilho \& Medronho.

O conceito do aplicativo é o de realizar cálculos de acordo com a equação e variáveis determinadas pelos usuários. A abordagem da autora diferenciou-se dos demais trabalhos analisados nessa análise bibliométrica por se tratar de um aplicativo móvel para realização de cálculos com apresentação de resultados para o usuário, e não um simulador ou laboratório virtual.

O trabalho de Lima (2019) foi abordado em dois artigos posteriores, publicados no XXXIX Congresso Brasileiro de Sistemas Particulados (Lima \& Santos, 2019 a,b), mostrando a relevância da dissertação e da pesquisa realizada pelos autores sobre o tema. 
Tabela 4: Artigos nacionais retirados do Google Scholar, ordenados por número de citações.

\begin{tabular}{|c|l|l|c|}
\hline Ordem & \multicolumn{1}{|c|}{ Autor } & \multicolumn{1}{|c|}{ Título } & \multicolumn{1}{|c|}{ Citações } \\
\hline \multirow{2}{*}{$1^{\circ}$} & LIMA \& SANTOS & $\begin{array}{l}\text { O uso de um aplicativo móvel para celular no ensino do projeto de } \\
\text { câmaras de separação gravitacional. }\end{array}$ & 2 \\
\cline { 2 - 4 } $3^{\circ}$ & SILVA et al. & $\begin{array}{l}\text { Desenvolvimento de um Software educativo para projeto da separação } \\
\text { sólido-líquido em centrífugas tubulares }\end{array}$ & 0 \\
\cline { 2 - 5 } $4^{\circ}$ & PATRÍCIO et al. & $\begin{array}{l}\text { Proposta de simulação dinâmica de colunas PSA (Pressure Swing } \\
\text { Adsorption) para treinamento de operadores. }\end{array}$ & 0 \\
\cline { 2 - 5 } $5^{\circ}$ & LIMA & $\begin{array}{l}\text { Desenvolvimento de um aplicativo híbrido para projetar equipamentos de } \\
\text { separação sólido-fluido no campo gravitacional e no campo centrífugo. }\end{array}$ & 0 \\
\cline { 2 - 5 } $6^{\circ}$ & BELISÁRIO et al. & $\begin{array}{l}\text { Relatos de experiência de inserção de tecnologias digitais no ensino de } \\
\text { Engenharia. }\end{array}$ & 0 \\
\hline
\end{tabular}

Fonte: Autores.

Silva et al. (2020) desenvolveram um software chamado o "Projeto Centrífuga", como ferramenta alternativa ao ensino de projeto de centrifugas tubulares. O software foi desenvolvido por alunos do curso de Engenharia Química da Universidade Federal do Triângulo Mineiro, no contexto da Disciplina de Operações Unitárias. O software foi posteriormente testado em sala de aula. Todo os alunos que utilizaram o software relataram que o uso do software agregou algum grau de conhecimento, sendo que $46,9 \%$ relataram que aprenderam muito com a atividade.

Patrício et al. (2016) analisaram a importância dos processos de produção e purificação de hidrogênio para fornecimento de combustíveis de baixo teor de enxofre, item utilizado na dessulfurização dos combustíveis, onde novas unidades para esses procedimentos têm sido expandidas ou implantadas. O trabalho objetivou a construção de um simulador que utilizasse dados obtidos em uma planta real para o processo de treinamento de operadores da indústria que operam com o sistema PSA (Pressure Adsorption Swing), utilizado na purificação de hidrogênio. Para isso, desenvolveram uma modelagem matemática para o processo de purificação de hidrogênio por adsorção em coluna de recheio para a construção de uma unidade modular. Esta modelagem foi simulada com dados de uma planta de purificação de hidrogênio de processo industrial. Parâmetros como temperatura, pressão e velocidade do escoamento foram variados, com o objetivo de verificar e analisar o comportamento da simulação em comparação com o comportamento previsto pela teoria. Com os resultados satisfatórios, a unidade modular foi criada e conectada ao simulador comercial DYNSIM, por intermédio do Excel (dados de entrada como características do gás e de alimentação da coluna). O ciclo de etapas de operação do sistema da PSA foi controlado por uma ferramenta do simulador comercial chamada cenários. Dessa forma, o sistema opera com a alternância de pressão proposta no projeto do ciclo.

Belisário et al. (2020) analisaram que a situação da educação em Engenharia Química precisa ser ativa, centrada no estudante, estimulando-os a aprender, analisar e sintetizar, propondo soluções. Integrar as ferramentas tecnológicas no processo de aprendizagem torna-se fundamental para a evolução do ensino. No trabalho, os autores explanaram sobre o uso do LabOps dentro da Universidade Federal de Minas Gerais e como o uso de tecnologias digitais vem moldando o modo de se ensinar Operações Unitárias no curso de Engenharia Química.

Dentro do laboratório, várias tecnologias são empregadas, algumas para simples comunicação do grupo, outras para divulgação de aulas e materiais (facebook, youtube), além da criação de projetos visando automação e acesso remoto aos equipamentos. Dessa forma, como pontuam os autores, o aluno estará em contato com o equipamento real gerando um conhecimento aplicável, uma vez que em simuladores digitais o conhecimento produzido é baseado em situações e contextos de 
processos reais que consideram, porém, apenas condições ideais/teóricas ou falhas pré-programadas de processos industriais.

O fato da busca realizada pelo Google Scholar trazer artigos nacionais que não estão nas plataformas Scopus e Web of Science mostra a dificuldade dos pesquisadores brasileiros em divulgar seus trabalhos em um nível internacional. Isto é fato e ocorre pelos mais diversos motivos, desde a falta de apoio financeiro, tanto para financiar as pesquisas quanto para a divulgação em revistas internacionais. No entanto, mesmo com a estrutura precária, as pesquisas têm ocorrido. A revisão apresentada neste trabalho evidencia a necessidade do desenvolvimento de novas políticas educacionais e também a necessidade de aumentar as parcerias de pesquisa com outros países.

\section{Considerações Finais}

Por meio desta revisão bibliométrica, verificou-se o estado da arte do uso de tecnologias no campo educacional, principalmente nas disciplinas que envolvem operações unitárias e processos de separação, temas de enorme importância para a indústria química.

Importante a validação do modo como os trabalhos estão sendo conduzidos, tendo em vista a ampla possibilidade que as tecnologias digitais provém para a educação tradicional. Os estudos, em sua maioria, verificaram a necessidade de reinventar o modo de aprendizagem, baseado no aspecto de troca de informações unilateral professor-aluno em uma metodologia em que o aluno é criador, divulgador e personagem ativo dentro do seu processo de ensino.

Dentro do contexto do uso de tecnologias nos campos das Engenharias e principalmente no tema de operações unitárias e processos de separação, ressalta-se que as ferramentas de simulação e laboratórios virtuais, em realidade virtual e/ou aumentada ganham um foco maior, pela possibilidade de oferecer ao aluno um ambiente em que é possível aprender com seus erros e experimentações. Além disso, abordagens como disponibilização de aulas em plataformas digitais e ensino por vídeo e imagens ou uso de aplicativos para dispositivos móveis que propõem um aprendizado ubíquo também são importantes para compor o cenário dessa revolução tecnológica na educação.

Com o resultado do levantamento bibliométrico realizado neste estudo, observa-se a possibilidade de inovação com o desenvolvimento de ferramentas e aplicações tecnológicas voltadas para a educação e instrução de alunos nas disciplinas de operações unitárias. Além disso, estudos com relação a outros campos e disciplinas das Engenharias podem corroborar para a adoção de ferramentas tecnológicas como ativos e objetos de conhecimento dentro das salas de aula.

\section{Agradecimentos}

Os autores agradecem o suporte do Programa de Mestrado Profissional em Inovação Tecnológica da Universidade Federal do Triângulo Mineiro.

\section{Referências}

Almeida, N. P. \& Santos, K. G. (2020). Chemical Engineering Laboratory teaching using Project-based learning approach: gas adsorption using banana peel. Research, Society and Development, 9(3), e184932716. https://doi.org/10.33448/rsd-v9i3.2716

Almeida, N. P., Canhadas, M. C., Albertini, M. R. M. C., Santos, K. G. \& Vieira Neto, J. L. (2020). Solid-fluid separation in the gravitational field: Courseware generation using computational fluid dynamics simulation. Computer Applications in Engineering Education, cae.22327, 1-14, https://doi.org/10.1002/cae.22327

Aria, M., \& Cuccurullo, C. (2017). bibliometrix: An R-tool for comprehensive science mapping analysis. Journal of Informetrics, 11(4), 959-975.

Belisário, A. B., Faria, D. G., de Souza Chaves, D. H., de Almeida, G. M., \& Cardoso, M. (2020). Relatos de experiência de in serção de tecnologias digitais no ensino de Engenharia. Revista Docência do Ensino Superior, 10, 1-18.

Belarmino, L. M., Dias, M. A. M., Melo, A. C., Batista, D. E. O., Araújo, F. M., \& Ramos, T. S. (2020). Route and strategies for implementing active methodologies in a higher education institution. Research, Society and Development, 9(7), e532974293. https://doi.org/10.33448/rsd-v9i7.4293 
Boaroli, L., Spacek, A. D., Neto, J. M., Izidoro, C. L., Maestrelli, E., \& Ando, O. H. (2017). Data monitoring and hardware control for app android by Bluetooth communication for laboratory teaching in electrical engineering courses. IEEE Latin America Transactions, 15(1), 31-39.

Borreguero, A. M., Valverde, J. L., García-Vargas, J. M., \& Sánchez-Silva, L. (2019). Simulator-based learning in the teaching of chemical engineering. Computer Applications in Engineering Education, 27(5), 1267-1276.

Botelho, W. T., Marietto, M. D. G. B., Ferreira, J. C. D. M., \& Pimentel, E. P. (2016). Kolb's experiential learning theory and Belhot's learning cycle guiding the use of computer simulation in engineering education: A pedagogical proposal to shift toward an experiential pedagogy. Computer Applications in Engineering Education, 24(1), 79-88.

Calvo, L., \& Prieto, C. (2016). The teaching of enhanced distillation processes using a commercial simulator and a project-based learning approach. Education for Chemical Engineers, 17, 65-74.

Christo, M. M. S., de Resende, L. M. M., \& Kuhn, T. D. C. G. (2018). Por que os alunos de engenharia desistem de seus cursos-um estudo de caso. Nuances: estudos sobre Educação, 29(1), 154-168.

Cruz, J. R., Ramos, R., \& Rodrigues, W. B. (2015). Guideline para desenvolvimento de aplicativos educacionais mobile. In Workshop de Computação da Faccamp. Campinas/São Paulo.

De Carvalho, G. D. G., Sokulski, C. C., Da Silva, W. V., De Carvalho, H. G., De Moura, R. V., De Francisco, A. C., \& Da Veiga, C. P. (2020). Bibliometrics and systematic reviews: A comparison between the Proknow-C and the Method Ordinatio. Journal of Informetrics, 14(3), 101043. https://doi.org/10.1016/j.joi.2020.101043

De Faria, J. W. V., Teixeira, M. J., Júnior, L. D. M. S., Otoch, J. P., \& Figueiredo, E. G. (2016). Virtual and stereoscopic anatomy: when virtual reality meets medical education. Journal of neurosurgery, 125(5), 1105-1111.

Fregolente, L., Venturelli, H., Rodrigues, J., Da Silva, E., Diniz, I., \& Maria, R. W. M. (2018). Project-Based Learning Applied to Distillation and Absorption Education: Integration Between Industry and a Chemical Engineering Undergraduate Course. Chemical Engineering Transactions, 69, 427-432.

Garcia-Zubia, J., Cuadros, J., Romero, S., Hernandez-Jayo, U., Orduna, P., Guenaga, M., \& Gustavsson, I. (2016). Empirical analysis of the use of the VISIR remote lab in teaching analog electronics. IEEE Transactions on Education, 60(2), 149-156.

Godoy, E. V., \& de Almeida, E. (2017). A evasão nos cursos de Engenharia e a sua relação com a Matemática: uma análise a partir do COBENGE. Educação Matemática Debate, 1(3), 339-361.

Henrique, J. P., de Sousa Jr, R., Secchi, A. R., Ravagnani, M. A., \& Costa, C. B. (2018). Optimization of chemical engineering problems with EMSO software. Computer Applications in Engineering Education, 26(1), 141-161.

Heradio, R., De La Torre, L., Galan, D., Cabrerizo, F. J., Herrera-Viedma, E., \& Dormido, S. (2016). Virtual and remote labs in education: A bibliometric analysis. Computers \& Education, 98, 14-38.

Juskeviciene, A., Jasute, E., Kurilovas, E., \& Mamcenko, J. (2016). Application of 1: 1 mobile learning scenarios in computer engineering education. International Journal of Engineering Education, 32(3), 1087-1096.

Köche, J. C. (2009). Fundamentos de metodologia científica: teoria da ciência e iniciação à pesquisa. In Fundamentos de metodologia científica: teoria da ciência e iniciação à pesquisa (pp. 182-182).

Li, X., \& Huang, Z. J. (2017). An inverted classroom approach to educate MATLAB in chemical process control. Education for Chemical Engineers, 19, 1-12.

Lima, G. M. D. (2019). Desenvolvimento de um aplicativo híbrido para projetar equipamentos de separação sólido-fluido no campo gravitacional e no campo centrífugo. Dissertação de Mestrado. Programa de Mestrado Profissional em Inovação Tecnológica, UFTM, Uberaba-MG.

Lima, G. M., \& Santos, K. G. (2019a). Desenvolvimento de um aplicativo móvel para projeto de separação sólido-gás empregando ciclones. In Anais do XXXIX Congresso Brasileiro de Sistemas Particulados-ENEMP, Belém, PA, Brasil.

Lima, G. M., \& Santos, K. G. (2019b). O uso de um aplicativo móvel para celular no ensino do projeto de câmaras de separação gravitacional. In Anais do XXXIX Congresso Brasileiro de Sistemas Particulados-ENEMP, Belém, PA, Brasil.

Mangucci, C. B., Stoppe, A. C. R., Morais, A. A., Melo, Y. A., Merola, G. N., \& Santos, K. G. (2020). Construction of a dust chamber didactic kit and computational fluid dynamics assessment: an active learning practice. Research, Society and Development, 9(11), e41691110069. 10.33448/rsd-v9i11.10069.

Martín-Gutiérrez, J., Mora, C. E., Añorbe-Díaz, B., \& González-Marrero, A. (2017). Virtual technologies trends in education. EURASIA Journal of Mathematics, Science and Technology Education, 13(2), 469-486.

Martín-Gutiérrez, J., Fabiani, P., Benesova, W., Meneses, M. D., \& Mora, C. E. (2015). Augmented reality to promote collaborative and autonomous learning in higher education. Computers in human behavior, 51, 752-761.

Mesquita, M. A. de, Mariz, F. B. de A. R., \& Tomotani, J. V. (2017). The Skateboard Factory: a teaching case on discrete-event simulation. Production, 27, e20162262. https://dx.doi.org/10.1590/0103-6513.226216

Monteiro, B. S., Gomes, A. S., \& Neto, F. M. M. (2016). Youubi: Open software for ubiquitous learning. Computers in Human Behavior, 55, $1145-1164$.

Moraes, E. P., Confessor, M. R., \& Gasparotto, L. H. (2015). Integrating mobile phones into science teaching to help students develop a procedure to evaluate the corrosion rate of iron in simulated seawater. Journal of Chemical Education, 92(10), 1696-1699.

Pagani, R. N., Kovaleski, J. L., \& Resende, L. M. (2015). Methodi Ordinatio: a proposed methodology to select and rank relevant scientific papers encompassing the impact factor, number of citation, and year of publication. Scientometrics, 105(3), 2109-2135. 
Research, Society and Development, v. 10, n. 11, e39101119155, 2021

(CC BY 4.0) | ISSN 2525-3409 | DOI: http://dx.doi.org/10.33448/rsd-v10i11.19155

P. N. A. Barata, M. R. Filho and M. V. A. Nunes (2015), Consolidating Learning in Power Systems: Virtual Reality Applied to the Study of the Operation of Electric Power Transformers. IEEE Transactions on Education, 58(4), 255-261, 10.1109/TE.2015.2393842.

Patricio, P. C. P. (2016). Proposta de simulação dinâmica de colunas PSA (Pressure Swing Adsorption) para treinamento de operadores. Dissertação de Mestrado. apresentada ao Programa de Pós-Graduação do Departamento de Engenharia Química da Escola de Engenharia da Universidade Federal de Minas Gerais.

Pereira, A. S., Shitsuka, D. M., Pereira, F. J., \& Shitsuka, R. (2018). Metodologia do trabalho científico. UFSM.

Potkonjak, V., Gardner, M., Callaghan, V., Mattila, P., Guetl, C., Petrović, V. M., \& Jovanović, K. (2016). Virtual laboratories for education in science, technology, and engineering: A review. Computers \& Education, 95, 309-327.

Qu, X., Zhang, R., Liu, B., \& Li, H. (2017). An improved TLBO based memetic algorithm for aerodynamic shape optimization. Engineering Applications of Artificial Intelligence, 57, 1-15.

Roman, C., \& García-Morales, M. (2019). Achieving a better understanding of binary azeotropic mixtures distillation through Aspen Plus process simulations. Computer Applications in Engineering Education, 27(6), 1453-1464.

Rodríguez, M., Díaz, I., Gonzalez, E. J., \& González-Miquel, M. (2018). Motivational active learning: An integrated approach to teaching and learning process control. Education for Chemical Engineers, 24, 7-12.

Santos, K. G., Silva, A, A. L., Toigo, S., Lima, L. J., Oliveira, J., Minaré, M. M., Martins, P. R. G., Dantas, S. C.; Silvério, B. C. (2021). Online blended approach (PBL) applied to learning separation process in chemical engineering. Research, Society and Development, 10(1), e30110111408, 2021. 10.33448/rsdv10i1.11408.

Silva, L. D., Lima G. M. Silvério, B.C. \& dos Santos, K. G. (2020). Desenvolvimento de um Software educativo para projeto da separação sólido-líquido em centrífugas tubulares. Revista Iberoamericana De Tecnología En Educación Y Educación En Tecnología, (25), e9. https://doi.org/10.24215/18509959.25.e9

Sotelino, E. D., Natividade, V., \& Travassos do Carmo, C. S. (2020). Teaching BIM and Its Impact on Young Professionals. Journal of Civil Engineering Education, 146(4), 05020005

Sung, Y. T., Chang, K. E., \& Liu, T. C. (2016). The effects of integrating mobile devices with teaching and learning on students' learning performance: A metaanalysis and research synthesis. Computers \& Education, 94, 252-275.

Udugama, I. A., Gernaey, K. V., Taube, M. A., \& Bayer, C. (2020). A novel use for an old problem: The Tennessee Eastman challenge process as an activating teaching tool. Education for Chemical Engineers, 30, 20-31. https://doi.org/10.1016/j.ece.2019.09.002

Viegas, C., Pavani, A., Lima, N., Marques, A., Pozzo, I., Dobboletta, E., \& Lima, D. (2018). Impact of a remote lab on teaching practices and student learning. Computers \& Education, 126, 201-216.

Zydney, J. M., \& Warner, Z. (2016). Mobile apps for science learning: Review of research. Computers \& Education, 94, 1-17. 